\title{
Validación de una Escala para evaluar Clima Escolar en estudiantes Universitarios $(\mathrm{ECE}-\mathrm{U})^{16}$
}

\author{
Alejandra García Arista \\ Magister en Psicología. \\ Universidad Nacional Autónoma de México, UNAM, Ciudad de México \\ Correo electrónico: platz83@hotmail.com \\ Patricia Andrade Palos \\ Doctora en Psicología \\ Universidad Nacional Autónoma de México, UNAM, Ciudad de México \\ Correo electrónico: p.andradepalos@gmail.com \\ Nazira Calleja \\ Doctora en Psicología \\ Universidad Nacional Autónoma de México, UNAM, Ciudad de México \\ Correo electrónico: ncalleja@unam.mx
}

Recibido: 22/01/2021 Evaluado: $18 / 06 / 2021$ Aceptado: 23/07/2021

\section{Resumen}

El objetivo del estudio fue analizar las características psicométricas de una escala para medir clima escolar en estudiantes universitarios. Se elaboró un instrumento de auto reporte con 61 reactivos de las áreas social, escolar y física del clima escolar contenidas en las dimensiones: clima académico, comunidad, seguridad y entorno institucional. Participaron 329 estudiantes universitarios (69\% mujeres y $31 \%$ hombres) con una edad promedio de 21.58 años, quienes de forma voluntaria accedieron a responder el cuestionario en línea. La muestra se dividió aleatoriamente en dos submuestras, 159 participantes para el análisis factorial exploratorio y 170 participantes para el análisis factorial confirmatorio. Se obtuvo un modelo con seis factores y el instrumento final se compuso de 21 reactivos con un valor de consistencia interna $\alpha=.858$. Esta escala es útil para medir clima escolar y tomar decisiones que impacten positivamente en los jóvenes universitarios.

Palabras clave

Comunidad escolar, clima académico, medición, entorno educativo, análisis factorial confirmatorio, educación superior.

16 Para citar este artículo: García, A., Andrade, P. y Calleja, N. (2022). Validación de una Escala para evaluar Clima Escolar en estudiantes Universitarios (ECE-U). Informes Psicológicos, 22(1), pp. 267-280. http://dx.doi.org/10.18566/infpsic.v22n1a16 


\title{
Validation of a Scale to evaluate School Climate in University students (ECE-U)
}

\begin{abstract}
The study seeks to analyze the psychometric characteristics of a scale to measure school climate in university students. A self-report instrument was developed with 61 items from the social, school and physical areas of the school climate contained in the following dimensions: academic climate, community, safety and institutional environment. 329 university students participated (69\% women and $31 \%$ men) with an average age of 21.58 years, who voluntarily agreed to answer the online questionnaire. The sample was randomly divided into two subsamples, 159 participants for the exploratory factor analysis and 170 participants for the confirmatory factor analysis. A model with six factors was obtained and the final instrument was composed of 21 items with an internal consistency value of $\alpha=.858$. This scale showed to be useful to measure school climate and to make decisions that positively impact university students.
\end{abstract}

\section{Keywords}

School community, academic climate, measurement, educational environment, confirmatory factor analysis, higher education.

\section{Validação de Escala para Avaliação do Clima Escolar em Estudantes Universitários (ECE-U)}

\section{Resumo}

0 objetivo do estudo foi analisar as características psicométricas de uma escala para medir 0 clima escolar em estudantes universitários. Foi elaborado um instrumento de autorrelato com 61 itens das áreas social, escolar e física do clima escolar contidos nas dimensões: clima acadêmico, comunidade, segurança e ambiente institucional. Participaram 329 estudantes universitários (69\% mulheres e $31 \%$ homens) com idade média de 21,58 anos, que aceitaram responder voluntariamente ao questionário online. A amostra foi dividida aleatoriamente em duas subamostras, 159 participantes para a análise fatorial exploratória e 170 participantes para a análise fatorial confirmatória. Obteve-se um modelo com seis fatores e 0 instrumento final foi composto por 21 itens com valor de consistência interna $\alpha=0,858$. Escala útil para medir o clima escolar e tomar decisões que impactam positivamente os universitários.

\section{Palavras chave} comunidade escolar, clima acadêmico, medição, ambiente educacional, análise fatorial confirmatória, ensino superior. 


\section{ntroducción}

La etapa universitaria implica una serie de desafíos como la adquisición de conocimientos y habilidades, lidiar con nuevas relaciones interpersonales e involucrarse en el funcionamiento de la universidad, entre otros; además, la forma como cada estudiante universitario percibe su entorno, es decir, el clima escolar, es muy importante porque influye en su rendimiento académico (Bradshaw, Waasdorp, Debnam \& Johnson, 2014).

Si bien muchos estudios en las últimas décadas abordan el concepto de clima escolar, aún existe variación en su definición; la mayoría de los investigadores coinciden en que el clima escolar se percibe de manera grupal, pues implica las interacciones entre la comunidad estudiantil compuesta por estudiantes, profesores y el personal administrativo (Juárez-Herrera, 2014; National School Climate Council, 2007).

\section{Multidimensionalidad del clima escolar}

Se ha considerado que el clima escolar es un constructo multidimensional porque las escuelas son organizaciones complejas compuestas por múltiples subsistemas que interactúan entre sí (Bryk, 2010) e incluye normas, objetivos, valores, relaciones interpersonales así como el entorno físico (National School Climate Council, 2007; Thapa, Cohen, Guffey \& Higgins-D'Alessandro, 2013).
Wang y Degol (2016) identificaron en su revisión teórica con 327 fuentes bibliográficas que el clima escolar se puede englobar en cuatro dimensiones: a) clima académico, b) comunidad, c) seguridad y d) ambiente institucional, lo que corrobora la multidimensionalidad del constructo. Por su parte, Kutsyuruba, Klinger y Hussain (2015) también analizaron la multidimensionalidad del clima escolar y propusieron tres grandes áreas: A) social, B) académica y C) física, las cuales abarcan las dimensiones y dominios propuestos por otros autores (Benbenishty, Astor, Roziner \& Wrabel, 2016; Lamoreaux \& Sulkowski, 2020; Wang \& Degol, 2016), como se puede apreciar en la descripción que se hace a continuación:

\section{A) Área social del clima escolar.}

Se refiere a la comunicación e interacción entre los miembros de la escuela, así como a los sentimientos y actitudes compartidos entre ellos (González-Benito et al., 2018). Como parte del área social del clima escolar se han estudiado las dimensiones de comunidad y seguridad; la primera enfatiza la calidad de las relaciones interpersonales dentro de la escuela y, según la revisión teórica de Wang y Degol (2016), engloba cuatro dominios: 1) calidad de las relaciones entre compañeros, 2) conectividad escolar, 3) respeto a la diversidad y 4) asociación comunitaria.

De acuerdo con algunos autores (Benbenishty et al., 2016), la seguridad implica tres dominios: 1) seguridad física, 2) emocional y 3) orden y disciplina. Con el incremento en el uso de las tecnologías de la información y comunicaciones, la seguridad también se ha estudiado en medios 
digitales ya que el ciberacoso es cada vez más frecuente (Holfeld \& Baitz, 2020).

\section{B) Área académica del clima escolar.}

El clima académico -diferente del clima escolar-, se enfoca en la calidad general del ambiente académico y engloba tres dominios: 1) liderazgo, 2) enseñanzaaprendizaje y 3) desarrollo profesional para profesores y personal (Wang \& Degol, 2016). Por su parte, Bryk (2010) ha resaltado la capacidad del profesor para brindar soporte y ser una guía en los procesos de enseñanza-aprendizaje e incluye la efectividad e influencia del profesor.

\section{C) Área física del clima escolar.}

Se refiere al entorno institucional, es decir, a los aspectos organizativos, físicos y materiales de la escuela. Algunos estudios (Lamoreaux \& Sulkowski, 2020; Kutsyuruba et al., 2015) han reconocido que las condiciones de infraestructura y el mobiliario de la escuela e incluso del salón de clase, pueden influir en la calidad del ambiente escolar.

\section{Medición del clima escolar}

La medición del clima escolar implica un desafío por su multidimensionalidad (Shukla et al., 2019) con notables diferencias en su operacionalización. A pesar de esta diversidad, Wang y Degol (2016) señalan que el 92\% de los estudios empíricos evalúan el clima escolar a través de encuestas de autoinforme. Además, la mayoría de los instrumentos se han dirigido a estudiantes de nivel básico y medio (Aldridge \& Ala'l, 2013; Byrd, 2017). Una de las propuestas pioneras fue la Escala de Clima Social EscolarCES de Moos y Trickett (1974), siendo uno de los cuestionarios más utilizados para medir clima escolar hasta nuestros días; otra escala muy usada actualmente es la Delaware School Climate SurveyStudent (DSCS-S) y se fundamenta en que un clima escolar saludable se caracteriza por dos elementos en equilibrio: a) apoyo o grado de reacción, esto por parte de los adultos y b) estructura o exigencia en cuanto a las normas y supervisión de la conducta de los estudiantes (Holst, Weber, Bear \& Lisboa, 2016).

Una escala elaborada en idioma español es la Escala del Clima Social Escolar (ECLIS), instrumento chileno que evalúa la percepción de fortalezas y debilidades en relación con cuatro áreas: 1) profesores, 2) compañeros, 3) satisfacción con la infraestructura y 4) satisfacción con la escuela como institución (Aron, Milicic \& Armijo, 2012).

En México, Juárez (2019) generó la Escala de Experiencia Universitaria, compuesta por cuatro factores: 1) satisfacción con la experiencia, 2) percepción de apoyo, 3) percepción de la experiencia y 4) ajuste al estilo de vida; aunque esta escala es de las pocas que evalúa vivencias universitarias, no incluye en su totalidad las dimensiones que componen el constructo de clima escolar, por ejemplo, la seguridad física y socioemocional escolar y las relacionadas con las reglas escolares (Cohen, McCabe, Michelli \& Pickeral, 2009; Thapa et al., 2013).

El clima escolar es una variable cada vez más estudiada, sin embargo, 
su conceptualización y operacionalización aún no es consistente entre autores (Kutsyuruba et al., 2015); además, la mayoría de las investigaciones se han centrado en estudiantes de nivel básico y medio (Torres, Estrada, García \& Rojas, 2017; United Nations Educational, Scientific and Cultural Organization [UNESCO], 2013), generando que la medición del clima escolar como constructo en menores de edad implique forzosamente el involucramiento de los padres (Grazia \& Molinari, 2020; Moore, Astor \& Benbenishty, 2020), dejando de lado las características de los estudiantes universitarios, en donde ya no es necesaria la participación familiar directa. Cabe mencionar que el concepto de clima del campus es el que se ha tratado de establecer como el equivalente a clima escolar en el nivel de educación superior, sin embargo, este constructo se enfoca principalmente en interacciones interculturales y raciales que no son aplicables a todos los contextos (Campbell \& Brauer, 2021; Lewis \& Shah, 2021). Ante la escasez de instrumentos para medir clima escolar en universitarios y con base en la revisión de la literatura internacional, el objetivo de este estudio es constituir, analizar y obtener las características psicométricas de un instrumento de clima escolar para estudiantes universitarios.

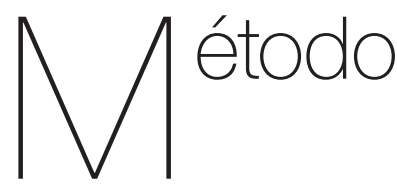

Se hizo un estudio no experimental, transversal, es decir, no se manipuló ninguna variable, solo se midieron en un momento determinado.

\section{Participantes}

Se contó con una muestra no probabilística de 329 estudiantes universitarios mexicanos (69\% mujeres y 31\% hombres) voluntarios, con edades de 18 a 28 años $(M=21.58, D E=2.44)$ que cursaban de primero hasta el último grado del nivel licenciatura, provenientes de instituciones públicas y privadas (85 y 15\% respectivamente).

\section{Instrumento}

Se definió el clima escolar como el conjunto de percepciones generadas por el entorno escolar, que considera elementos académicos, sociales y físicos del ambiente institucional. Para constituir el instrumento de este trabajo, se tomaron en cuenta las tres áreas del clima escolar (académica, social y física) y por cada área se incluyeron dimensiones que comúnmente se han definido como indispensables. Así del área académica se midió el clima académico, del área social las dimensiones de comunidad y seguridad y del área física se incluyó el entorno institucional (Aldridge \& McChesney, 2018; Cohen et al., 2009; Wang \& Degol, 2016).

Para la elaboración del banco de reactivos de la Escala de Clima Escolar para Universitarios (ECE-U) se retomaron reactivos de tres instrumentos que correspondían a las dimensiones propuestas: la Escala de Experiencia Universitaria (Juárez, 2019), la Delaware School Climate Survey-Student (DSCS-S) (Bear, Gaskins, Blank \& Chen, 2011) y la Escala de Clima Social Escolar (ECLIS) (Aron et al., 2012); se decidió utilizar reactivos de estos instrumentos por su idoneidad para 
la etapa universitaria, su construcción y/o adaptación a población hispanohablante y su amplio uso para evaluar el constructo de clima escolar.

La escala generada es autoaplicable en dos formatos en versión impresa o vía electrónica, no existen diferencias significativas entre los formatos ya que el contenido de los reactivos y opciones de respuesta son los mismos, aplicándose el instrumento de forma virtual para este estudio. Se redactaron 61 reactivos que abarcaron las siguientes dimensiones: clima académico, comunidad, seguridad y entorno institucional; además, las opciones de respuesta son en escala tipo Likert de siete puntos y van de totalmente de acuerdo a totalmente en desacuerdo. Se consideraron siete opciones de respuesta debido al nivel educativo de los participantes y con la finalidad de obtener mejores propiedades psicométricas.

\section{Procedimiento}

La escala se publicó en redes sociales y se realizaron aplicaciones en aulas virtuales, enfatizando el otorgamiento del consentimiento y la participación voluntaria. Una vez recopilados los datos, se analizaron las propiedades psicométricas del instrumento con el sistema estadístico SPSS-23. Las pruebas de reactivos se realizaron con la muestra total; posteriormente, ésta se dividió aleatoriamente en dos submuestras de 159 participantes (113 mujeres y 46 hombres, con edades de entre 18 y 28 años; $M=21.54, D E=$ 2.42) para el análisis factorial exploratorio y análisis de consistencia interna y 170 participantes (115 mujeres y 55 hombres, con edades de entre 18 y 28 años; $M=$ 21.62, $D E=2.46$ ) para el análisis factorial confirmatorio realizado con el programa estadístico AMOS 24.

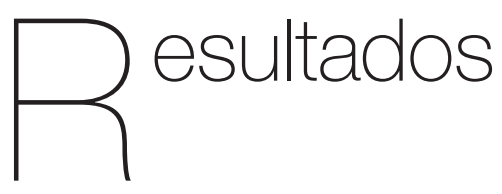

Inicialmente se realizó un análisis de los 61 reactivos (sesgo, curtosis y variabilidad de las respuestas), considerando los criterios recomendados por Vázquez (2012) que sugieren una curtosis menor a 10 y la asimetría inferior a 3 como condiciones prácticas para evaluar la normalidad de una variable. Al finalizar estos procedimientos estadísticos, quedaron 48 reactivos para continuar con los análisis. Posteriormente, se realizó un análisis factorial exploratorio con el método de extracción de mínimos cuadrados no ponderados con rotación oblicua como la oblimin, con normalización kaiser y se estimó la correlación reactivo-total corregida. Se utilizó la rotación oblicua porque se considera que las dimensiones están relacionadas y forman parte de un mismo constructo.

Para conformar los factores se contemplaron, al menos, tres reactivos por factor, cada reactivo con saturación $\geq .40$ en un solo factor (estructura factorial simple), correlación reactivo-total corregida $\geq .20$, congruencia conceptual reactivofactor, y consistencia interna por factor $\geq .60$ calculada por el Alfa de Cronbach. El análisis factorial exploratorio arrojó 22 reactivos agrupados en seis factores con una varianza total explicada de 55.36\% $(\mathrm{KMO}=.819$, Barlett X2 $=1454.711, \mathrm{p}<$ .001). La consistencia interna global fue de $\alpha=.858$ (ver Tabla 1). 
Tabla 1

Características factoriales y de confiabilidad de la ECE-U

\begin{tabular}{llcl}
\hline \multicolumn{2}{l}{ Factor 1 : Soporte del profesor } & Peso Factorial \\
\hline 8. Los profesores saben corregir sin que los es- & .768 & \\
$\quad$ tudiantes se sientan humillados & .798 & Varianza explicada $=35.32 \%$ \\
9. $\quad$ Los profesores tratan a todos los alumnos por igual & .837 & .726 \\
10. Los profesores aceptan opiniones diferentes a las suyas & Los profesores se preocupan por sus alumnos & \\
11.
\end{tabular}

Factor 2 : Pertenencia universitaria

\begin{tabular}{lll}
\hline 14. Entrar a la Universidad fue un orgullo para mí & .683 & \\
15. Me gusta mi vida escolar & .674 & Varianza explicada $=8.214 \%$ \\
16. Recuerdo que al ingresar a la Universidad me sentía contento & .787 & \\
20. Me gusta mi Universidad y eso me hace sentir bien & .449 &
\end{tabular}

Factor 3 : Relación con compañeros

$\begin{array}{lcc}\text { 23. La paso bien con mis compañeros de clase } & .771 & \\ \text { 26. Me siento respetado por mis compañeros } & .670 & \text { Varianza explicada }=11.672 \% \\ \text { 31. Confío en mis compañeros } & .584 & \\ \text { 32. Mis compañeros son amigables entre ellos } & .819 & \end{array}$

\section{Factor 4 : Agresividad escolar}

37. Mis compañeros son muy agresivos .679

38. A mis compañeros les gusta poner sobrenombres

39. Mis compañeros se han burlado de $m$

.782

40. Los estudiantes amenazan e intimidan a otros

.715

Factor 5 : Reglamentación universitaria

45. En la universidad está claro lo que está permitido y lo que está prohibido

.976

.731

Varianza explicada $=25.19 \%$

46. Los estudiantes saben cuáles son las reglas de la Universidad

.446 ción de violencia sexual y/o de género
Varianza explicada $=17.46 \%$ .715

Factor 6 : Recursos institucionales

\begin{tabular}{llll}
\hline 53. Los baños de mi escuela regularmente están limpios & .700 & \\
55. Dispongo de suficiente material de trabajo en el salón & .545 & Varianza explicada $=9.12 \%$ \\
56. Dispongo de suficientes libros en la biblioteca & .721 & \\
\hline
\end{tabular}

Posteriormente se realizó el análisis factorial confirmatorio con la segunda submuestra y se probó un modelo con los 6 factores como variables latentes y los reactivos como variables observadas. Se realizaron las pruebas de adecuación muestral y un análisis factorial confirmatorio de máxima verosimilitud (ML). Las varianzas de las variables latentes se fijaron en 1.0 y las varianzas de los términos de error fueron especificadas como parámetros libres. 
Se realizaron los ajustes con base en los criterios estadísticos (índices de modificación y saturación factorial de cada reactivo) y teóricos (coherencia conceptual reactivo-factor) con el fin de mantener el valor conceptual del instrumento. Los ajustes se hicieron de modo secuencial, se eliminó el reactivo 15, y se asociaron algunos errores según los valores de la covarianza estandarizada (ver Figura 1). El modelo mostró un ajuste adecuado con las seis dimensiones.

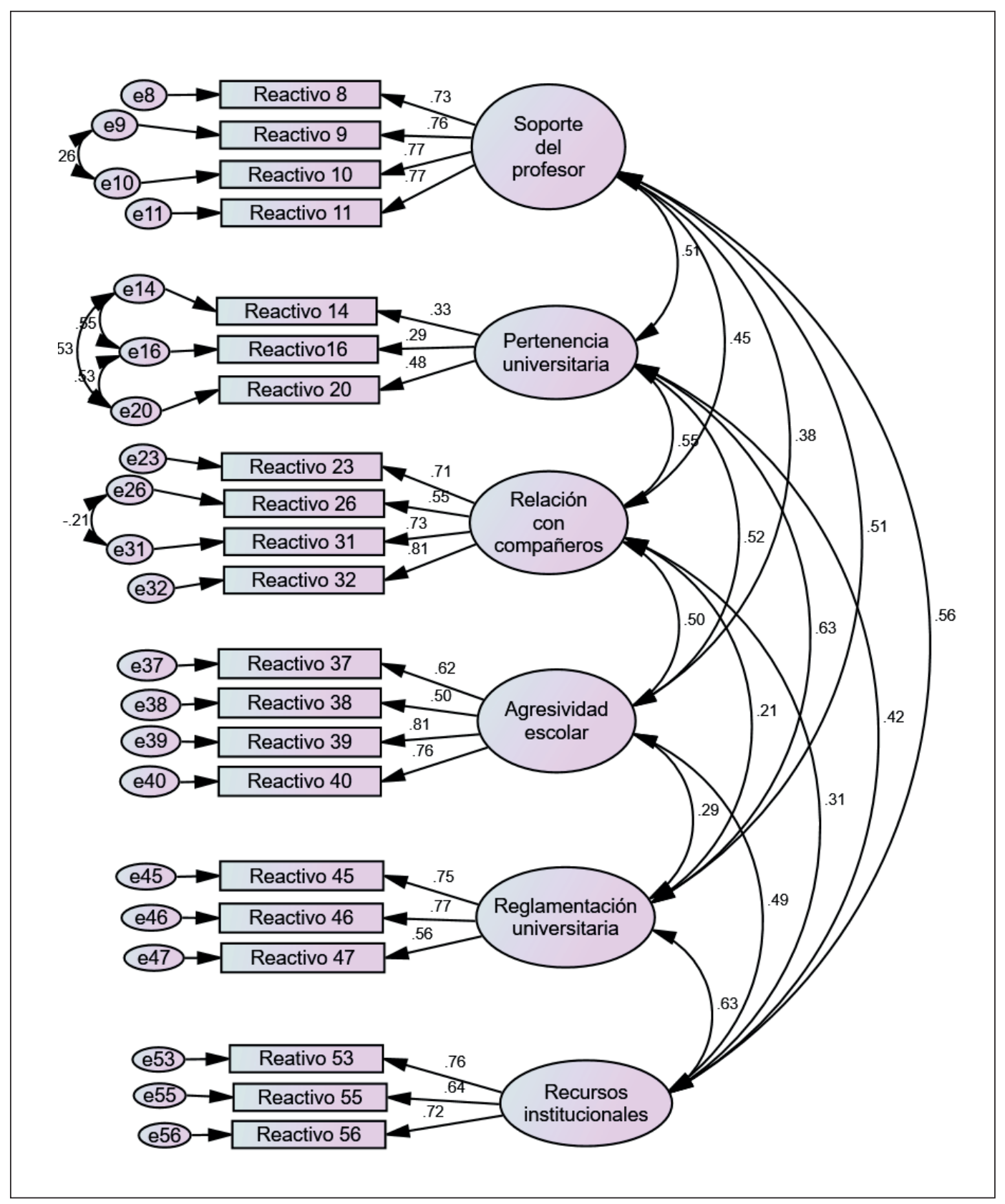

Figura 1. Modelo del Análisis Factorial Confirmatorio de la ECE-U 
Idealmente, $\Delta \chi^{2}$ no debería ser significativo, $y$ en el presente modelo el valor obtenido fue de $\Delta \chi^{2}=.00$, sin embargo, este valor es sensible al tamaño de la muestra, por lo que se consideraron los otros índices para respaldar la bondad del modelo; los indicadores de bondad de ajuste para el modelo se muestran en la Tabla 2. En resumen, el modelo final de la escala presentó niveles aceptables de ajuste a los datos, siendo consistente con el análisis exploratorio.

Tabla 2.

Índices de ajuste para el Análisis Factorial Confirmatorio de la ECE-U

\begin{tabular}{cccccccc}
\hline Modelo & \multicolumn{8}{c}{ Índices de ajuste } \\
\hline & $\chi^{2}$ & gl & $\chi^{2 / g l}$ & GFI & CFI & TLI & RMSEA \\
\hline ECE-U & $260.59^{*}$ & 179 & 1.456 & .912 & .935 & .924 & .052 \\
\hline
\end{tabular}

Nota: ${ }^{*} p<.01$

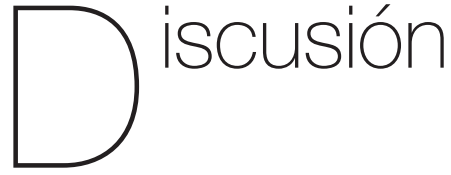

Existe una cantidad considerable de propuestas para evaluar el clima escolar, principalmente en los niveles de educación básico y medio; sin embargo, deben incrementarse los esfuerzos por unificar la composición del constructo ante su multidimensionalidad. En este estudio inicialmente se propuso una escala que contenía las tres áreas del clima escolar distribuidas en cuatro dimensiones, sin embargo, la composición final de la escala se distribuyó en seis factores; a pesar de esta redistribución cada una de estas dimensiones contiene elementos que han sido considerados como necesarios para operacionalizar el clima escolar (Cohen et al., 2009).

El análisis factorial confirmatorio reveló que la estructura factorial de seis dimensiones esperada se ajusta a los datos, siendo semejante a la obtenida en población mexicana de educación secundaria en el reporte de Caso, Díaz, Chaparro y
Urias (2011), quienes obtuvieron en su escala cinco factores: 1) relación con profesores, 2) relación entre alumnos, 3) violencia dentro del plantel, 4) disciplina escolar y 5) condiciones físicas del plantel. Estos factores se identificaron en la construcción de la escala de este estudio, además del factor de pertenencia universitaria; esto puede deberse a que en la Universidad es más importante para los estudiantes identificarse con su institución educativa y sentirse miembro de la comunidad universitaria porque es una etapa donde los jóvenes ya eligieron su vocación y seleccionaron la institución educativa en donde desean cursar su formación profesional, acorde a sus intereses y aptitudes, mientras que, en el nivel básico o medio superior, las escuelas pueden ser seleccionadas aún por otros criterios como la cercanía con el domicilio o trabajo de los padres.

En gran medida, el clima académico está dado por el clima social escolar, por lo que el elemento de comunidad resulta particularmente relevante, ya que las universidades, al igual que cualquier centro educativo, se han convertido en algo más que edificios y entornos de aprendizaje 
académico, favoreciendo también el desarrollo de habilidades de socialización e identificación. Además, existe evidencia de que el apoyo del profesor resulta una variable importante para que los alumnos perciban un buen clima escolar (Aron et al., 2012); tanto es así que, en el instrumento propuesto en este estudio, fue el factor que mejores propiedades psicométricas presentó. Estos resultados concuerdan con lo encontrado por Zullig, Koopman, Patton y Ubbes (2010), quienes identificaron la relación positiva entre estudiantes y maestros como uno de los dominios más importantes del clima escolar.

Otro factor importante fue el de relación con compañeros, pues los jóvenes aún se encuentran en la adolescencia, la etapa del desarrollo en la que los pares son un agente social relevante. Así, estas relaciones influyen también en factores emocionales y motivacionales para aprender (Eccles et al., 1993) y deben considerarse como parte de las prácticas de enseñanza, ya que representan elementos clave para el aprendizaje.

Es importante enfatizar que las relaciones entre estudiantes y profesores, a su vez, resultan elementos indispensables para evaluar el sentido de pertenencia (Knekta, Chatzikyriakidou \& McCartney, 2020), lo cual no es extraño pues formar parte de un grupo y tener sentido de pertenencia social son necesidades humanas básicas que se han relacionado positivamente con mayor salud mental y menos síntomas de ansiedad, depresión y estrés (Thompson, Wood \& Davis, 2019).

En este sentido, en diversas ocasiones se ha mencionado la importancia de la disminución de la agresividad escolar y la implementación de reglas escolares para el funcionamiento psicológico saludable y mayor aprendizaje (Holfeld \& Baitz, 2020; Moore et al., 2020). En la construcción de este instrumento se propusieron algunos reactivos que evaluaban la seguridad en medios tecnológicos, sin embargo, para la población evaluada no fueron elementos relevantes y fueron eliminados antes de ser incluidos en el análisis exploratorio; posiblemente, por eso la mayoría de los instrumentos que miden clima escolar se enfocan en las relaciones cara a cara (Aldridge \& Ala'l, 2013; Aron et al., 2012).

Los recursos institucionales también se han relacionado con un clima escolar positivo, se ha señalado la necesidad de poner a disposición los recursos de aprendizaje necesarios para favorecer las actividades de enseñanza-aprendizaje (Lenz, Rocha \& Aras, 2020); sin embargo, también se ha evidenciado la importancia de la disponibilidad y accesibilidad de las instalaciones sanitarias para el adecuado desempeño académico de los estudiantes (Yakubu, 2017). Resulta relevante que muchos de los reactivos iniciales que se eliminaron después de los análisis psicométricos del instrumento, tenían contenido relacionado con infraestructura de la universidad; esto puede deberse a que, si bien los participantes de este estudio pertenecen a universidades públicas de la Ciudad de México y zonas metropolitanas, éstas cuentan con financiamiento gubernamental suficiente para cubrir recursos institucionales básicos, no siendo así en otras universidades de zonas menos favorecidas.

La escala propuesta en este estudio resulta un instrumento confiable y pertinente, ofrece a los investigadores y 
profesionales interesados en la evaluación del clima escolar un cuestionario breve y válido para estudiantes universitarios. También resulta útil para evaluar la efectividad de los programas de intervención dirigidos a mejorar diversas áreas escolares, a saber: a) la convivencia entre los miembros de las universidades, por ejemplo entre alumnos y profesores; b) la seguridad escolar, como programas para prevenir la agresividad escolar; y c) el conocimiento de la reglamentación universitaria y, en general, el agrado de los estudiantes con la institución universitaria, ya que se ha observado que la percepción favorable del clima escolar se ha relacionado con resultados positivos tanto académicos, como sociales y emocionales (Bear et al., 2011).

Respecto a las limitaciones de este estudio se pueden mencionar las siguientes: los datos se recopilaron mediante un muestreo no aleatorio, todas las aplicaciones de los cuestionarios se realizaron en línea, además de que la mayoría de los estudiantes pertenecían a escuelas públicas, lo que limita la generalización de los resultados a otros estudiantes, por ejemplo, a los de instituciones privadas. Otra limitación es no haber tenido grupos homogéneos entre las áreas de estudio que permitieran realizar comparaciones del clima escolar entre jóvenes que estudian en diversas áreas del conocimiento. En próximos estudios se sugiere ampliar el tamaño de la muestra, además de evaluar si existen diferencias en las dimensiones del clima escolar en alumnos de semestres iniciales y finales, incluso realizar un estudio longitudinal donde se evalúe el clima escolar en jóvenes que inician la educación superior y realizar un seguimiento en distintos momentos, para poder evaluar los cambios en este constructo a través del tiempo. También sería importante evaluar el clima escolar en alumnos de posgrado e incluir en el instrumento la percepción no solo de los alumnos sino también de los profesores o trabajadores universitarios.

Otra alternativa para definir el clima escolar sería a través de entrevistas en las cuales los alumnos y profesores indicaran qué aspectos son los más importantes que ellos consideran que componen ese clima que favorece la permanencia de los alumnos en la universidad, así como su rendimiento académico.

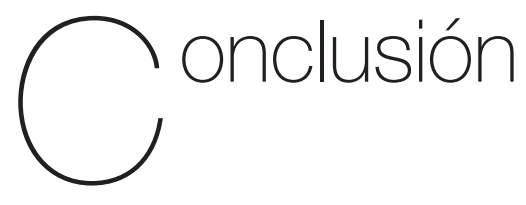

La evaluación del clima escolar es necesaria para tomar decisiones que impacten de manera positiva en los jóvenes. Aunque se cumplió el objetivo de este estudio y se logró la propuesta de un instrumento de clima escolar para estudiantes universitarios con propiedades psicométricas adecuadas, además de ser breve, se sugiere llevar a cabo más investigación en torno al clima escolar para comprender la asociación temporal entre los factores que la componen y la generalización a otras muestras.

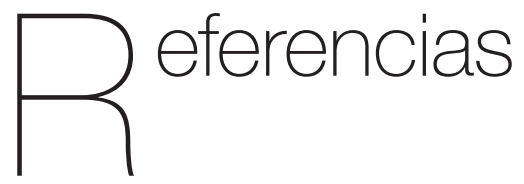

Aldridge, J. \& Ala'l, K. (2013). Assessing students' views of school climate: 
Developing and validating the What's Happening in This School? (WHITS) questionnaire. Improving Schools, 16(1), 47-66. doi: 10.1177/1365480212473680

Aldridge, J. M. \& McChesney, K. (2018). The relationships between school climate and adolescent mental health and wellbeing: $A$ systematic literature review. International Journal of Educational Research, 88, 121145. doi: 10.1016/j.ijer.2018.01.012

Aron, A. M., Milicic, N. \& Armijo, I. (2012). Clima social escolar: una escala de evaluación -Escala de Clima Social Escolar, ECLIS. Universitas Psychologica, 11(3), 803-813. Recuperado de http://www.scielo.org.co/ scielo.php?script=sci_arttext\&pid=S165792672012000300010\&lng=en\&tlng=.

Bear, G., Gaskins, C., Blank, J. \& Chen, F. (2011). Delaware School Climate SurveyStudent: Its factor structure, concurrent validity, and reliability. Journal of School Psychology, 49(2), 157-174. doi: 10.1016/j. jsp.2011.01.001

Benbenishty, R., Astor, R., Roziner, I. \& Wrabel, S. (2016). Testing the causal links between school climate, school violence, and school academic performance: a cross-lagged panel autoregressive model. Educational Researcher, 45(3), 197-206. doi: 10.3102/0013189X16644603.

Byrd, C. M. (2017). The complexity of school racial climate: Reliability and validity of a new measure for secondary students. British Journal of Educational Psychology, 87(4), 700-721. doi: 10.1111/bjep.12179.

Bradshaw, C. P., Waasdorp, T. E., Debnam, K. J. \& Johnson, S. L. (2014). Measuring school climate in high schools: A focus on safety, engagement, and the environment.
Journal of School Health, 84(9), 593-604. doi: 10.1111/josh.12186

Bryk, A. S. (2010). Organizing schools for improvement. Phi Delta Kappan, 91(7), 23-30. doi: 10.1177/003172171009100705

Campbell, R. \& Brauer, M. (2021). Is discrimination widespread? Testing assumptions about bias on a university campus. Journal of Experimental Psychology: General, 150, 756-777. doi: 10.1037/xge0000983

Caso, J., Díaz, C., Chaparro, A. \& Urias, E. (2011). Propiedades psicométricas de las escalas, cuestionarios e inventarios de la Estrategia Evaluativa Integral 2010: Factores asociados al aprendizaje (UEE Reporte Técnico 11-002). Recuperado de http: //uee.uabc.mx

Cohen, J., McCabe, L., Michelli, N. M. \& Pickeral, T. (2009). School climate: Research, policy, practice, and teacher education. Teachers College Record, 111(1), 180-213. Retrieved from https:// www.researchgate.net/profile/JonathanCohen-11/publication/235420504_ School_Climate_Research_Policy_ Teacher_Education_and_Practice/ links/59d67f050f7e9b42a6aa0145/ School-Climate-Research-PolicyTeacher-Education-and-Practice.pdf

Eccles, J. S., Wigfield, A., Midgley, C., Reuman, D., Maclver, D. \& Feldlaufer, H. (1993). Negative effects of traditional middle schools on students' motivation. Elementary School Journal, 93(5), 553574. doi: 10.1086/461740

González-Benito, A., Pacheco, M., Guadix, I., Belmonte, O., López, A., Losoviz, L. \& Casanovas, S. (2018). Guía para la 
mejora del clima escolar en los centros educativos. Guías de educación en derechos y ciudadanía global de Unicef. Comité español. Recuperado de https:// www.unicef.es/educa/biblioteca/guiamejora-clima-escolar-centros-educativos

Grazia, V. \& Molinari, L. (2020). The multidimensional school climate questionnaire (MSCQ) parent-version: Factorial structure and measurement invariance, International Journal of School \& Educational Psychology, 8(3), 1-5. doi: 10.1080/21683603.2020.1828205

Holfeld, B. \& Baitz, R. (2020). The mediating and moderating effects of social support and school climate on the association between cyber victimization and internalizing symptoms. Journal of Youth and Adolescence: A Multidisciplinary Research Publication, 49, 2214-2228. doi: 10.1007/s10964-020-01292-0

Holst, B., Weber, J., Bear, G. G. \& Lisboa, C. (2016). Adaptación transcultural y validación del contenido del Delaware School Climate Survey- Student (DSCS-S) en Brasil. RELIEVE. Revista Electrónica de Investigación y Evaluación Educativa, 22(2), 1-12. doi: 10.7203/relieve.22.2.6459

Juárez, A. (2019). Depresión en jóvenes universitarios: una aproximación desde el modelo ecológico de conductas de salud. (Tesis doctoral). Universidad Nacional Autónoma de México.

Juárez-Herrera, M. (2014). Estudio exploratorio del clima escolar universitario (Tesis doctoral). Universidad Iberoamericana, México.

Kutsyuruba, B., Klinger, D. A. \& Hussain, A. (2015). Relationships among school climate, school safety, and student achievement and well-being: A review of the literature. Review of Education, 3(2), 103-135. doi: 10.1002/rev3.3043

Knekta, E., Chatzikyriakidou, K. \& McCartney, M. (2020). Evaluation of a questionnaire measuring university students' sense of belonging to and involvement in a biology department. Life Sciences Education, 19(3), 1-14. doi: 10.1187/cbe.19-09-0166

Lamoreaux, D. \& Sulkowski, M.L. (2020). An alternative to fortified schools: Using crime prevention through environmental design (CPTED) to balance student safety and psychological well-being. Psychological School, 57, 152-165. doi: 10.1002/ pits. 22301

Lenz, A.S., Rocha, L. \& Aras, Y. (2020). Measuring school climate: A systematic review of initial development and validation studies. International Journal for the Advancement of Counselling, 43, 48-62. doi: 10.1007/s10447-020-09415-9

Lewis, R. \& Shah, P. (2021). Black students' narratives of diversity and inclusion initiatives and the campus racial climate: An interest-convergence analysis. Journal of Diversity in Higher Education, 14, 189202. doi: $10.1037 /$ dhe0000147

Moore, H., Astor, R. A. \& Benbenishty, R. (2020). Role of school-climate in schoolbased violence among homeless and non-homeless students: Individual- and school-level analysis. Child Abuse \& Neglect, 102(2), 104378. doi: 10.1016/j. chiabu.2020.104378

Moos, R. \& Trickett, E. (1974). Classroom environment scale manual. Palo Alto: Consulting Psychologist Press. 
National School Climate Council (2007). The school climate challenge: Narrowing the gap between school climate research and school climate policy, practice guidelines and teacher education policy. Retrieved from http://www.schoolclimate.org/ publications/policy-briefs.php

Shukla, K. D., Waasdorp, T. E., LindstromJohnson, S., Orozco-Solis, M. G., Nguyen, A. J., Rodríguez, C. C. \& Bradshaw, C. P. (2019). Does school climate mean the same thing in the United States as in Mexico? A focus on measurement invariance. Journal of Psychoeducational Assessment, 37(1), 55-68. doi: 10.1177/0734282917731459

Thapa, A., Cohen, J., Guffey, S. \& HigginsD'Alessandro, A. (2013). A review of school climate research. Review of Educational Research, 83(3), 357-385. doi: 10.3102/0034654313483907

Thompson, K., Wood, D. \& Davis, Mc.N. (2019). Sex differences in the impact of secondhand harm from alcohol on student mental health and university sense of belonging. Addictive Behaviors, 89, 57-64. doi: 10.1016/j.addbeh.2018.09.012.

Torres, C., Estrada, M., García, M.T. \& Rojas, H. (2017). Clima escolar e involucramiento parental en dos muestras de escolares en educación básica, la percepción de los alumnos, un estudio exploratorio. Revista Electrónica de Psicología Iztacala, 20(1), 1-18. Recuperado de https://www. medigraphic.com/pdfs/epsicologia/epi2017/epi171a.pdf
United Nations Educational, Scientific and Cultural Organization (2013). Análisis del clima escolar: ¿Poderoso factor que explica el aprendizaje en América Latina y el Caribe? Chile: Santillana.

Vázquez, J. (2012). Modelo de ecuaciones estructurales en psicología. (Tesis de maestría). Universidad Politécnica de Valencia, España. Recuperado de https://riunet.upv.es/bitstream/ handle/10251/44523/Vazquez_Molina_ Joan_TFM_Investmat.pdf?sequence=1

Wang, M. \& Degol, J. (2016). School climate: a review of the construct, measurement, and impact on student outcomes. Educational Psychology Review, 28, 315352. doi: 10.1007/s10648-015-9319-1

Yakubu, S. (2017). Space for convenience planning and academic performance of secondary school students in Oyo state, Nigeria. Journal of Education and Practice, 8(13), 73-78. Retrieved from de https:// files.eric.ed.gov/fulltext/EJ1143955.pdf

Zullig, K. J., Koopman, T. M., Patton, J. M. \& Ubbes, V. A. (2010). School climate: historical review, instrument development, and school assessment. Journal of Psychoeducational Assessment, 28(2), 139-152. doi: 10.1177/0734282909344205 